\title{
Extension problem of complex line bundles for Kähler surfaces
}

\author{
By \\ Shigeo NaKano
}

\section{§1. Introduction.}

The purpose of this note is to prove the following

Theorem. Let $\widetilde{\omega}: \mathscr{M} \rightarrow S$ be a complex analytic family of compact Kähler surfaces over a complex analytic space $S$. Let 0 be a point of $S$ and $M_{0}=\widetilde{\omega}^{-1}(0)$. Suppose there is given a complex line bundle $B_{0}$ over $M_{0}$, then there exists a neighborhood $S^{\prime}$ of 0 on $S$, such that the set

$$
\left\{s \in S^{\prime} \mid B_{0} \text { can be extended to } M_{s}\right\}
$$

is an analytic set in $S^{\prime}$.

As for the definition of an analytic family parametrized by a complex analytic space, we follow Kuranishi [2]. Similar extension problem has recently been treated by $\mathrm{Ph}$. A. Griffiths (Proceedings of the Conference on complex analysis, Minneapolis 1964), and the author was influenced by his work. But our result is not covered by his, and the author thinks it suitable to give here a presentation independent of his. Our treatment goes along the line of Nakano [4].

\section{\$2. The case of Kuranishi family.}

Let $\pi: \mathscr{M}^{\prime} \rightarrow T$ be the locally complete family of deformations with centre $\pi^{-1}(0)=M_{0}^{\prime}=M_{0}$, constructed by Kuranishi in [2]. Then $T$ is an analytic set in a neighborhood of 0 in a complex Euclidean 
space and $\mathscr{M}^{\prime}$ is a complex analytic space whose underlying topological space is $\times T$. (Here $\mathbb{M}$ denotes the differentiable manifold underlying $M_{t}^{\prime}, t \in T$ ).

$\mathbb{M}$ is covered by open sets $\{U\}$, and in $U \times T$ there are two complex valued $C^{\infty}$ functions $\zeta^{1}$ and $\zeta^{2}$ which, for each fixed $t$, form a local coordinate system for the complex structure $M_{t}^{\prime}$. If we take analytic local coordinates $\left(z^{1}, z^{2}\right)$ on $U$ for the structure $M_{0}^{\prime}$, then $\zeta^{\lambda}=g^{\lambda}(z, t)$, where $g^{\lambda}$ is differentiable on $U \times T$ and holomorphic in t. (See Lemma 1.2 of Kuranishi [3]).

To discuss the extension of a complex line bundle $B_{0}$ over $M_{0}^{\prime}$, we stand on a viewpoint taken in Nakano [4]. We choose a family of Kähler structures on $\left\{M_{t}^{\prime}\right\}_{t \in T}$ and we can state the condition:

"Let $c$ be the Chern class of $B_{0}$, considered in the complex valued cohomology group of $\mathbb{R}$, then $B_{0}$ can be extended to $M_{t}^{\prime}$ if and only if $c$ is of type $(1,1)$ in the Kähler structure of $M_{t}^{\prime}$."

$c$ is represented by a real, closed differential form $\Phi$ on $R$, and since it is real, $\Phi$ gives a cohomology class of type $(1,1)$ for structure $t$ if and only if $\int_{M_{t}^{\prime}} \wedge \Phi=0$ for any holomorphic differential form $\Psi$ on $M_{t}^{\prime}$ of degree 2.

Because $M_{t}^{\prime}$ are Kähler, $\operatorname{dim}_{c} H^{0}\left(M_{t}^{\prime}, \Omega_{t}^{2}\right)$ is independent of $t$. This fact, combined with the theorem of Grauert on coherency of direct images of coherent sheaves (Grauert [1]), shows that there exists a family of bases $\left\{\Psi_{1}(, t), \cdots, \Psi_{q}(, t)\right\}$ for $H^{0}\left(M_{t}^{\prime}, \Omega_{t}^{2}\right)$, depending holomorphically on $t$. (The last phrase means that, when $\Psi_{r}(, t)$ is expressed in terms of local coordinates $(\zeta)$, then the coefficients are holomorphic in $t$.)

Put $u_{r}(t)=\int_{\mathbb{M}} \Psi_{r}(\zeta, t) \wedge \Phi(z) \quad(r=1, \cdots, q)$, then $u_{r}(t)=$ $\int_{M} \Psi_{r}(g(z, t), t) \wedge \Phi(z)$ are clearly holomorphic functions on $T$, and we see $B_{0}$ can be extended to $M_{t}^{\prime}$ if and only if $u_{r}(t)=0$ for $r=1, \cdots, q$. This proves the theorem for our particular case. 


\section{§3. General case.}

Given a complex line bundle $B_{0} \rightarrow M_{0}$ and an analytic family $\widetilde{\omega}: \mathscr{M} \rightarrow S$ of deformations of $M_{0}=\widetilde{\omega}^{-1}(0)$, we can find a neighborhood $S^{\prime}$ of 0 in $S$ and a holomorphic map $\tau: S^{\prime} \rightarrow T$ such that $\tau(0)=0$, and $\mathscr{M}$ is induced by $\tau$ from the universal family $\mathscr{H}^{\prime} \rightarrow T$. (Because of Kähler property, $\operatorname{dim} H^{1}\left(M_{t}^{\prime}, \Theta_{t}\right)$ is independent of $\left.t\right)$. It is clear that $B_{0}$ can be extended to $M_{s}$ if and only if $B_{0}$ can be extended to $M_{\tau(s)}^{\prime}$. Hence our theorem is valid for general case too.

\section{§. Remarks。}

If $M_{0}$ is a projective surface, then our result shows that the set of those projective surfaces of the family, which are embedded in a projective space by deformations of the given projective embedding of $M_{0}$, forms an analytic set in the parameter space $S^{\prime}$. It is desirable to decide if similar result holds for the set of all projective surfaces of the family. In this connection the author would like to raise the following question:

In an analytic family $\widetilde{\omega}: \mathscr{M} \rightarrow S$ of compact complex manifolds, is it possible that there is an analytic family $\mathscr{B}$ of complex line bundles except over $V_{0}$, such that it cannot be extended to $V_{0}, V_{0}$ still being projective?

It is also desirable to extend our result to the family of manifolds of arbitrary dimension.

\section{$\mathbb{R E F E R E N C E ~}$}

[1] H. Grauert, Ein Theorem der analytischen Garbentheorie und die Modulräume komplexer Strukturen. Publications I. H. E. S., No. 5 (1960).

[2] M. Kuranishi, New proof for the existence of locally complete families of complex structures. Proc. of the Conference on Complex Analysis, Minneapolis 1964, 142-154, Springer Verlag.

[3] ( On the locally complete familes of complex analytic structures, Ann. of Math., Vol. 75 (1962), 536-577.

[4] S. Nakano, A condition for the extension of a complex line bundle for a family of Kähler surfaces. J. Math. Kyoto Univ., Vol. 2 (1963), 183-191. 
